\title{
A NOVEL FRAMEWORK FOR SIMULATION-BASED OPTIMISATION OF MAINTENANCE SYSTEMS
}

\author{
Alrabghi, A. ${ }^{*} \&$ Tiwari, A.* \\ * Department of Industrial Engineering, University of Jeddah, Jeddah, Saudi Arabia \\ ${ }^{* * *}$ Manufacturing Department, Cranfield University, Cranfield, United Kingdom \\ E-Mail: aalrabghi@uj.edu.sa, a.tiwari@cranfield.ac.uk
}

\begin{abstract}
The maintenance function in manufacturing has been gaining growing interest and significance. Simulation based optimisation has a high potential in supporting maintenance managers to make the right decisions in complex maintenance systems. Surveys in maintenance optimisation have repeatedly reported the need of a framework that provides adequate level of details to guide both academics and practitioners in optimising maintenance systems. The purpose of the current study is to address this gap by developing a novel framework that supports decision making for maintenance in manufacturing systems. The framework is developed by synthesising research attempts to optimise maintenance by simulation, examining existing maintenance optimisation frameworks and capturing framework requirements from review papers in the area as well as publications on future maintenance applications. As a result, the framework addresses current issues in maintenance such as complexity, multi-objective optimisation and uncertainty. The framework is represented by a standard flowchart to facilitate its use.

(Received in February 2015, accepted in July 2015. This paper was with the authors 1 week for 1 revision.)
\end{abstract}

Key Words: Simulation, Optimisation, Maintenance, Framework, Complex Systems

\section{INTRODUCTION}

In recent years, the maintenance function in manufacturing has been gaining growing interest and significance. Improving maintenance is seen as an investment that will have a positive impact on product quality, asset availability and asset productivity. Simulation-based optimisation has a strong potential in supporting maintenance managers to make the right decisions in complex maintenance systems [1].

Surveys such as that conducted by Alrabghi and Tiwari [1] and Horenbeek et al. [2] revealed that the approaches to optimise maintenance varied significantly in the literature. This includes a wide range of optimisation objectives, decision variables and optimisation algorithms. Moreover, very little was found in the literature on comparing and selecting the optimum maintenance strategy. Overall, these studies highlight the need for a framework that unifies the approach to optimising maintenance systems.

The main aim of this research is to develop a simulation-based multi-objective optimisation framework that supports decision making for maintenance in manufacturing systems. The proposed framework is a systematic approach detailing the steps required to successfully optimise simulated maintenance systems which would be helpful in directing future research. It can assist in displaying available options for a specific maintenance system as well as guiding both researchers and practitioners to determine which data are required to optimise the maintenance system.

\section{OVERVIEW OF EXISTING MAINTENANCE OPTIMISATION FRAMEWORKS}

Generic frameworks that guide the optimisation process are well established in the literature. For instance, Deb [3] identified 7 steps that are usually involved in an optimal formulation 
process (see Fig. 1). The first step is to ensure that optimisation is right for the problem in interest, whereas the four subsequent steps are focused on the formulation of the optimisation problem. This is followed by selecting a suitable optimisation algorithm based on the problem's characteristics and obtaining the solution. Likewise, other comparable general models that can be applied to optimise any engineering problem appear in the literature [4].

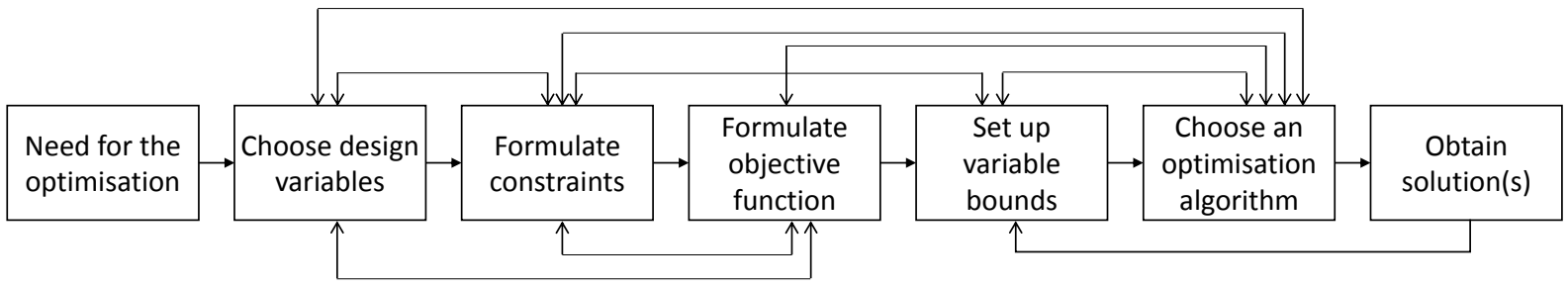

Figure 1: Flow chart of a general optimisation process [3].

However, few studies attempted to develop a framework for maintenance optimisation. Chien et al. [5] proposed a customised systematic approach for determining the optimal maintenance policy in automated manufacturing systems. As can be seen in Fig. 2, the approach utilises simulation, experimental design and regression metamodels. Hence it assumes that it is possible to construct a valid regression model which limits the applicability of the approach in complex problems.

\begin{tabular}{|c|c|c|c|c|c|c|}
\hline $\begin{array}{l}\text { Define the } \\
\text { problem }\end{array}$ & $\begin{array}{c}\text { Develop the } \\
\text { simulation model }\end{array}$ & $\begin{array}{l}\text { Determine the feasible } \\
\text { ranges of the } \\
\text { controllable variables }\end{array}$ & $\begin{array}{c}\text { Build the Taguchi } \\
\text { Experimental Design }\end{array}$ & $\begin{array}{c}\text { Perform simulation } \\
\text { runs and ANOVA }\end{array}$ & $\begin{array}{l}\text { Implement regressions } \\
\text { analysis and conduct } \\
\text { metamodel validation }\end{array}$ & $\begin{array}{l}\text { Formulate and solve } \\
\text { the decision model }\end{array}$ \\
\hline
\end{tabular}

Figure 2: Systematic approach for determining the optimal maintenance policy [5].

Riane et al [6] developed a graphical framework for simulation based maintenance which allows the modelling of a dynamic system and optimises the maintenance policy. As shown in Fig. 3, the framework begins with the modelling aspect to ensure the behaviour of the system is represented accurately. That is followed by simulating potential maintenance strategies and finally optimisation to obtain the solution. The framework is useful on the high-level. However, it does not provide detailed assistance to the user. For example, how to formulate the maintenance problem, how to decide which maintenance strategies are relevant or which optimisation algorithm is suitable.

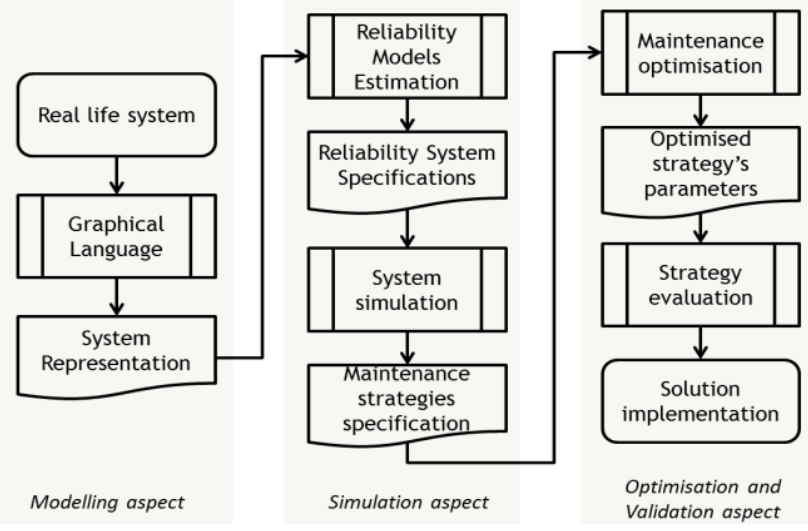

Figure 3: Decision making framework for maintenance problems; adapted from [6].

Horenbeek et al. [2] suggested a generic maintenance optimisation classification framework. It is a result of literature review aimed at collecting factors that have an impact on the optimisation model such as optimisation objectives and parameters. As shown in Fig. 4, it provides a general overview of all possible maintenance optimisation models making it 
possible to select the appropriate model. However, the user needs to be experienced in order to choose suitable modelling techniques, data sources, maintenance actions and maintenance policies amongst other options in the framework to fit the problem at hand. Horenbeek et al. [2] recognised the need for a decision structure that guides both practitioners and academics in implementing the right optimisation models with the available data while considering the specific business context; which is the purpose of this paper.

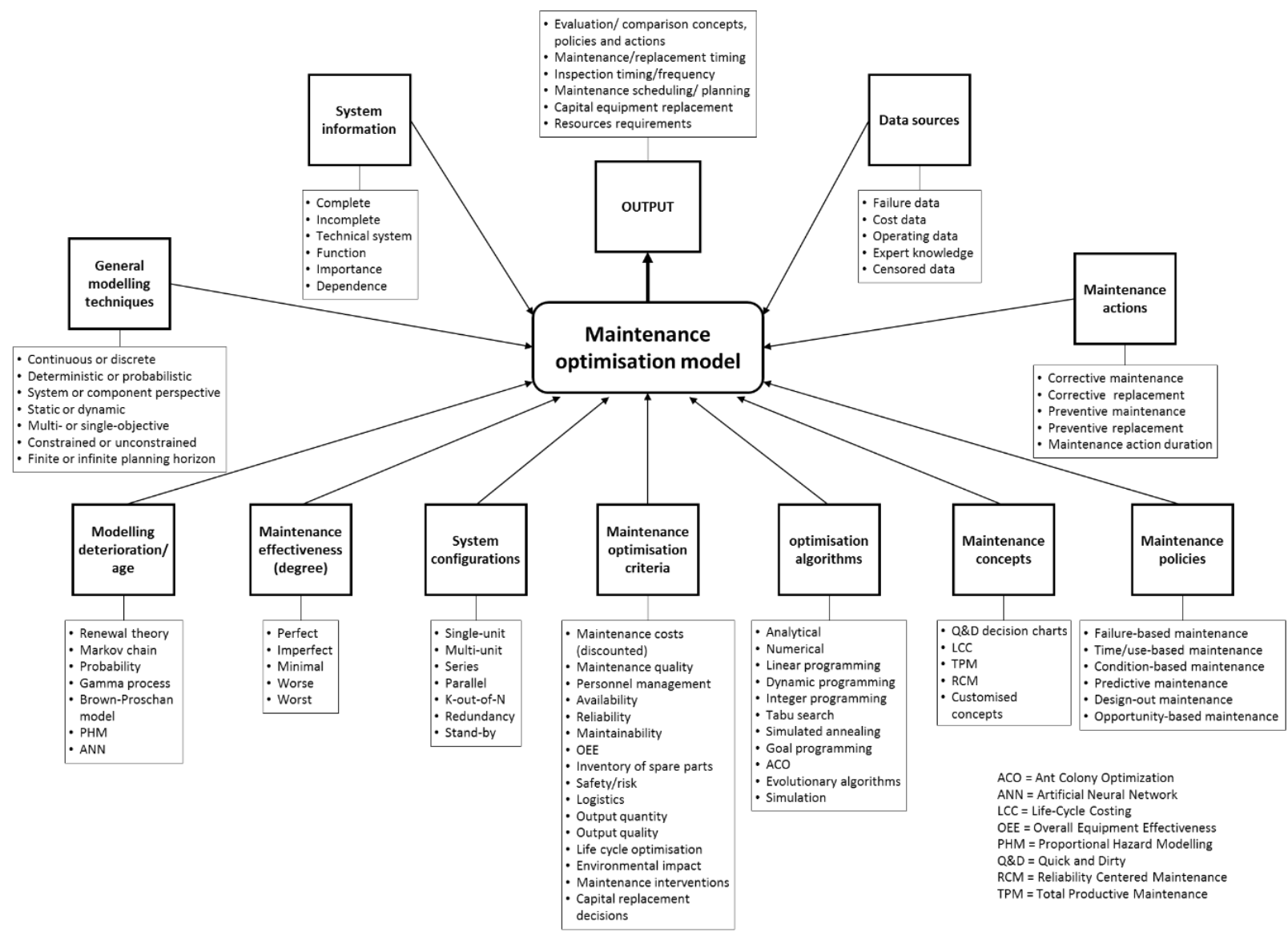

Figure 4: Maintenance optimisation classification framework [2].

Overall, present frameworks either lack the applicability to complex maintenance systems, do not provide the level of details needed for a typical practitioner or are not designed in a structure that could be followed to make decisions. The evidence presented in this section suggests that there is a need for a framework that supports academics and practitioners in optimising maintenance systems. The next section describes the research methodology for developing a framework for simulation-based optimisation of maintenance systems.

\section{RESEARCH METHODOLOGY}

Fig. 5 presents the methodology followed in order to develop a framework for simulation based optimisation for maintenance systems. The existing maintenance optimisation frameworks were investigated in order to build on its strengths and establish its limitations.

In order to locate review papers in maintenance optimisation, 34 publications were generated by searching in Scopus citation database for 'maintenance', 'optimisation' and 'review' in journal article titles and keywords while excluding papers in life or health sciences. Publications were refined by examining the titles and excluding those out of the study scope. In order to include papers published in other databases or those that did not use our search terms, citations in the review papers were traced. In total, ten relevant journal 
articles were incorporated [1, 2, 7-14]. Survey papers were examined paragraph by paragraph with specific focus on review findings, research gaps and limitations and recommendations for further research. Comments and critiques to the approaches researchers undertake when optimising maintenance systems were documented. Additionally, aspects that need to be considered in future research attempting to optimise maintenance systems were captured.

The need for a simulation based optimisation framework for maintenance systems

Document the framework requirements

- Maintenance-related requirements

- User-related requirements
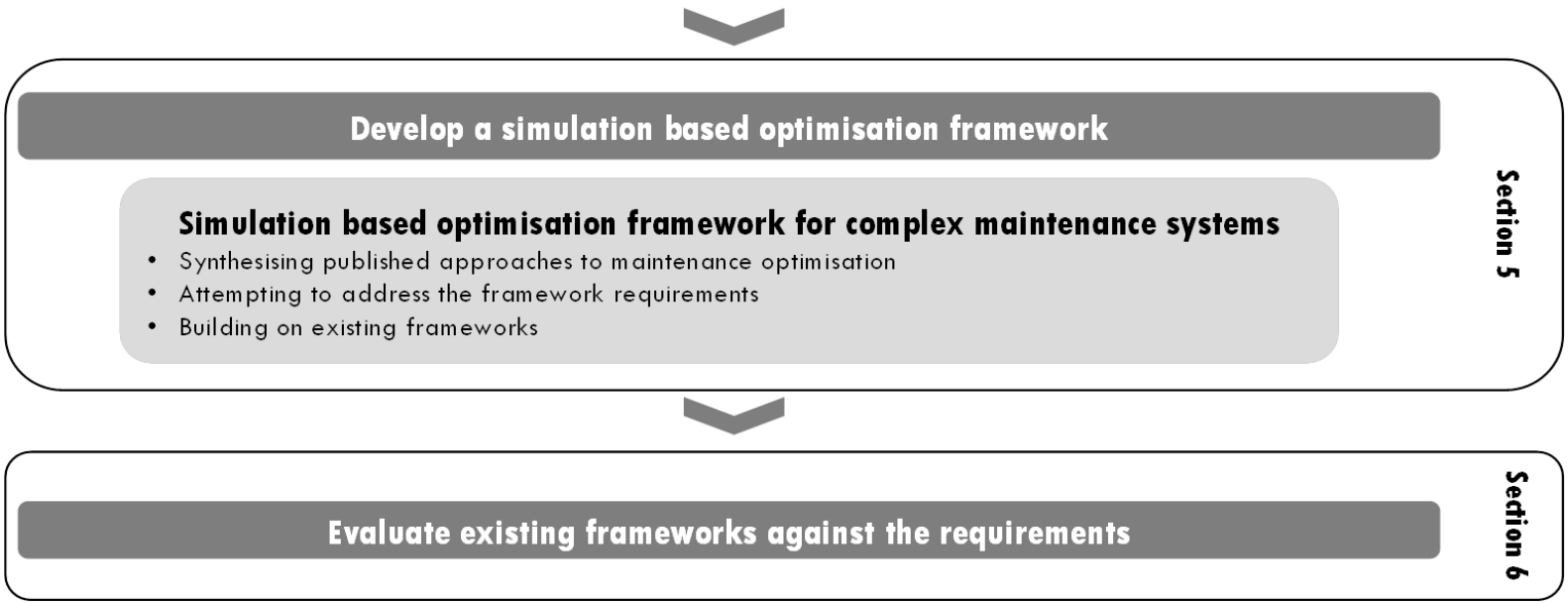

Figure 5: Framework development methodology.

In parallel, research papers on contemporary maintenance applications and upcoming trends were examined to ensure the framework addresses current and possible future challenges. We searched for the keywords 'prospective' or 'trends' or 'future', all in combination with 'maintenance' in the title, abstract or keywords of publications listed in the Scopus database. The search covered journal article titles while limiting the publications date to the last five years and excluding papers in life or health sciences to ensure only timely requirements are captured. To extend the set of relevant publications, reference lists in resulting papers were searched for related papers. In total, ten publications were identified [1, 10, 15-22].

Requirements relating to the simulation and modelling aspects were considered irrelevant as the current research assumes the availability of a valid simulation model of the maintenance system in interest. In addition, only papers related to maintenance in production setting were considered relevant thereby excluding papers considering maintenance in product service systems such as aviation [23] or power transformers [24]. 
Framework requirements were categorised into two types: user-related requirements and maintenance-related requirements. A novel framework was developed by synthesising published approaches to maintenance optimisation and attempting to meet all possible framework requirements. A standard flowchart tool was used to represent the framework due to its familiarity and ability to depict decision structures clearly. Finally, existing frameworks were evaluated to reveal how well they meet the requirements.

\section{THE FRAMEWORK REQUIREMENTS}

The requirements captured from survey papers in maintenance simulation optimisation as well as papers on future maintenance strategies and applications were grouped into userrelated requirements and maintenance-related requirements as follows:

\subsection{User-related requirements}

Requirement 1: Assist users with typical uncertainty found in maintenance systems.

A number of authors $[1,8,14,25]$ have reported that the availability of accurate data is a challenge in maintenance optimisation. In practical situations, it is almost always necessary to make assumptions or approximations. The proposed framework therefore has to advise the user on suitable strategies to deal with the typical uncertainty found in maintenance systems.

Requirement 2: Assist users to adapt maintenance models to their specific business needs. There is a large volume of published simulation optimisation studies in maintenance. However, the optimal problem formulation varies significantly [1, 8]. The framework has to make an attempt to synthesise the published studies and encompass all possible variations. It can then propose the most suitable parameters for the maintenance problem in hand including the objective functions, decision variables, constraints and optimisation algorithms. This will enable industrial companies to build optimisation models that meet their specific business needs.

Requirement 3: Enable users to solve multi-objective optimisation.

Traditionally, research in maintenance was investigating single objective optimisation problems only. Multi-objective optimisation is an under-explored area in maintenance optimisation $[2,22]$. Most engineering problems - including maintenance - require solving multiple objectives simultaneously [26]. The framework needs to allow the decision maker to solve multi-objective problems to provide flexibility in the increasingly dynamic manufacturing environment.

Requirement 4: Assist users with complex maintenance systems.

Maintenance systems are becoming increasingly complex including thousands of components with various dependencies between them [7]. It may not be possible to optimise all components or assets in the system. Therefore, the user requires assistance in defining the problem scope efficiently. Nevertheless, the optimisation problem may still be complex resulting in high computation expenses. Appropriate strategies will be required to reduce the computation time.

Requirement 5: An operational decision making tool suitable for maintenance managers and practitioners.

It has been suggested that most published maintenance optimisation models were developed in academia away from industry and real practises [2, 10, 14]. This led to many theoretical models that can perhaps be implemented in special cases only. Dekker [8] highlights the difficulty of understanding and interpreting maintenance optimisation models. Technicians, engineers and managers need a user-friendly approach to optimise their maintenance systems. The framework can make use of standardised methodologies that are known to the typical practitioner in the field [14]. In addition, the framework should provide sufficient guidance 
assuming the practitioner has no or little information on optimisation. This includes a standardised optimisation procedure in addition to instructions on how to correctly interpret the optimisation results. The typical problem user should be able to use the framework to support operational decision making.

\subsection{Maintenance-related requirements}

Requirement 6: Incorporating production dynamics and spare parts management.

A number of studies have examined systems that are inter-related with maintenance such as production dynamics and spare parts [27, 28]. They showed that these systems have a substantial effect on maintenance performance. Furthermore, optimising them jointly with maintenance can yield better results. The framework should consider the environment surrounding the maintenance system and allow the investigation of such important factors.

Requirement 7: Allow the investigation of several maintenance strategies simultaneously. There is little found in the literature on optimising several maintenance strategies simultaneously for the same asset [1]. Most researchers assume that a specific maintenance strategy is the optimum. Therefore, the research focus is on optimising the maintenance strategy parameters without investigating alternative strategies [13]. It is possible to have several maintenance strategies applicable for each asset in the system e.g. Preventive Maintenance (PM) and Condition Based Maintenance (CBM) or perhaps several variations of policies for the same strategy such as time-based PM and opportunistic PM. The framework should allow the investigation of more than one maintenance strategy yielding the optimum maintenance strategy and policy for each asset in the system.

Requirement 8: Incorporating possible future maintenance strategies.

Contemporary manufacturing systems are becoming increasingly complex which makes the task of predicting failures and intervening in the right time challenging. CBM aims to monitor the condition of an asset and trigger maintenance actions when deterioration occurs [18]. An advanced alternative strategy is designing self-maintenance machines where assets are able to monitor its health, diagnose faults and maintain its function [15]. It is a methodology that gained popularity recently in the literature. Additionally, it is expected to continue growing both in research and practice. The framework has to consider the possible future applications of CBM and self-maintenance.

Requirement 9: Integration with e-maintenance.

The framework would have to accommodate the growing interest in the concept of emaintenance. The ability of gaining remote access to the maintenance information infrastructure through various means, the integration of maintenance with other functions within the organisations, the enhanced collaboration opportunities and the utilisation of real time data to design optimum maintenance strategies are some of the potential benefits of emaintenance [16]. The framework can extend the use of e-maintenance platforms by advising a systematic and perhaps an automatic procedure to utilise the continuously streaming data and provide decision-making support in real time.

\section{A NOVEL FRAMEWORK FOR SIMULATION-BASED OPTIMISATION OF MAINTENANCE SYSTEMS}

This simulation based optimisation framework aims to support decision making for maintenance in manufacturing systems at the operational level. By providing a systematic procedure for conducting simulation optimisation to improve maintenance systems, it can assist in investigating available options for a specific maintenance system as well as guiding both researchers and practitioners in determining which data are required to implement the research. 
The framework on a high level is shown in Fig. 6. It takes the user through eight main steps that were mainly adapted from generic optimisation frameworks (see for example [3]). However, it is specifically developed for optimising complex maintenance models. Each main step is a sub-process that contains further instructions in a flow chart structure to provide detailed assistance to the user. The framework assumes that there is already a valid simulation model that represents the real maintenance system. The first seven main steps are conducted before engaging the optimisation engine whereas the last main step, namely decision making, is conducted after the optimisation results are obtained. The main contemporary issues in maintenance optimisation that are addressed are shown around the framework.

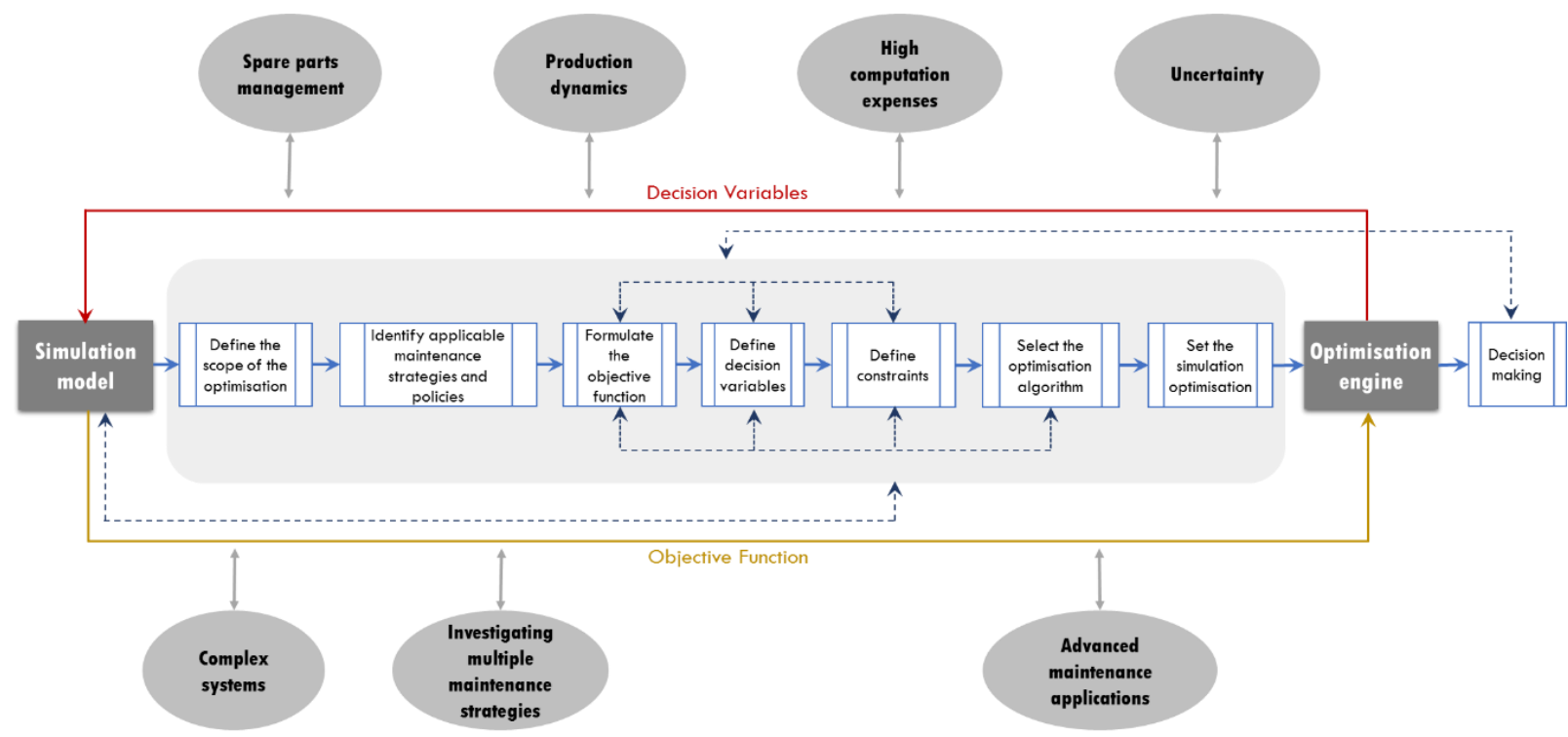

Figure 6: Simulation-based optimisation framework for maintenance systems on a high level.

In addition to the high level shown above, there are two more levels. The second level is shown in Fig. 7 whereas the third level details selected processes further. These sub-processes on the third level are shown in Appendix A.

A description of each main step is as follows:

1. Define the scope of the optimisation: As modern manufacturing systems are becoming more complex with many components interacting, it may not be practical to optimise all assets in the manufacturing system. An assessment can be conducted to identify the most critical assets. If the modelling level of details goes beyond assets to subsystems or components within assets then various tools can be utilised to identify the most critical subsystems/components such as Failure Modes and Effect Analysis (FMEA), operational data and expert and vendors experience. Defining the scope of optimisation also includes the decision of optimising other systems jointly with maintenance such as the production system and/or the spare parts management system. Optimising both systems with maintenance have shown to produce better results [1]. However, including more decision variables will inevitably increase the problem complexity. In addition, the inclusion/exclusion of a system in the optimisation should be affected by the user's ability to alter the decision variables.

2. Identify applicable maintenance strategies and policies: This step leads the user to investigate what maintenance strategies can be applied in the selected assets. This will depend on the available level of maintenance infrastructure such as skilled technicians and condition monitoring equipment. In addition, the production configuration might affect the range of possible maintenance strategies and policies. For instance, we might want to consider opportunistic PM in continuous production where shutdowns can be exploited [29]. Maintenance strategies are generally categorised into Corrective Maintenance (CM), PM or 
CBM. There are a number of policies within each strategy. For example, CBM can be inspection based or continuous monitoring based. In addition, self-maintenance is included as strategy to accommodate for the increasing interest and possible future applications [15]. In this step, the user can assign several maintenance strategies/policies for each asset. The framework will then identify the optimum maintenance strategy/policy and its parameters.

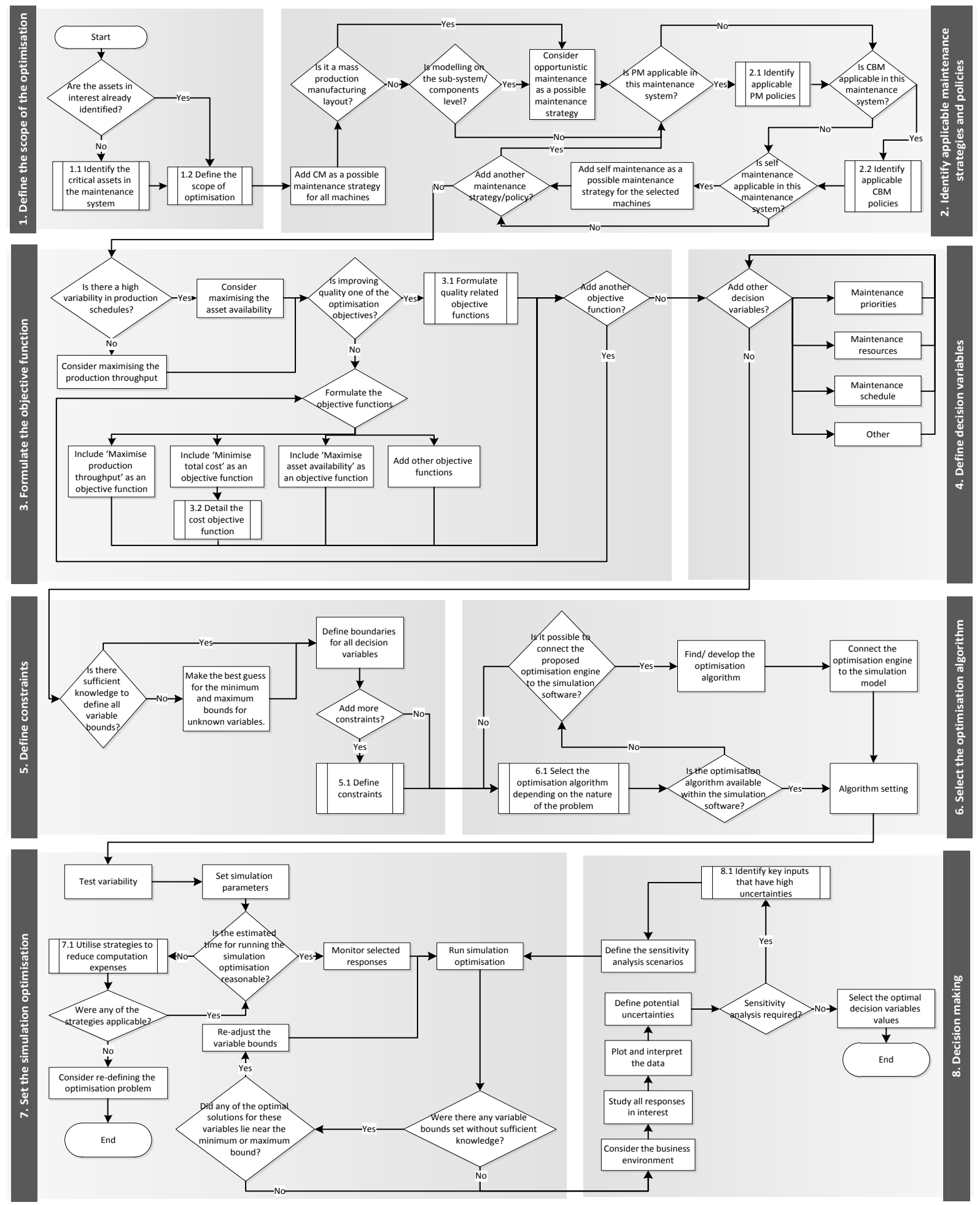

Figure 7: The second level of the framework.

3. Formulate the objective functions: Formulating the objective functions can be affected by production and demand patterns. For example, if there is high uncertainty in 
demand it might be worth considering maximising asset availability. This will ensure assets are more capable of handling fluctuations in production schedules. On the other hand, if uncertainty in production schedules is relatively low it might be worth considering maximising the production throughput. Some optimisation studies are conducted mainly to enhance quality measures. In such cases, objectives such as minimising cycle times and lead times can be included as objective functions. Although minimising cost is an objective in most maintenance optimisation studies [1], detailing the cost function varies widely and depends on several factors such as the defined scope of the optimisation (step 1) as well as the objective function. For example, if spare parts are jointly optimised with maintenance then costs associated with spare part policies need to be detailed. Researchers in maintenance have not treated multi-objective optimisation in much detail despite its significant advantages [1, 2]. This framework allows the user to optimise multiple objectives simultaneously.

4. Define the decision variables: Depending on the outcome of preceding steps, controlled variables can be defined. For example, PM strategies usually involve setting PM frequency as a decision variable whereas CBM usually involves setting inspection frequency and/or maintenance threshold as decision variables. In addition, the scope of the optimisation will have an effect on the choice of decision variables. For instance, if spare parts policies are optimised jointly with maintenance one will be interested in optimising the policy parameters such as maximum and minimum stock levels. Most of the decision variables are defined within previous steps in the framework to avoid adding decision nodes to recall the selected maintenance strategies or the optimisation scope. On the other hand, some decision variables are not related to outcomes from previous steps such as maintenance technicians, inspection equipment or maintenance priorities which can be defined in this step.

5. Define constraints: Technical knowledge can assist in defining feasible ranges for each variable. If the user is lacking the required knowledge, it may be necessary to make assumptions and redefine the ranges after conducting initial experiments [3]. In addition, the framework enables the user to define a range of constraints related to maintenance resources, maintenance schedule, spare parts, production, costs and other customised constrains.

6. Select the optimisation algorithm: This step includes choosing the optimisation algorithm and setting the appropriate algorithm parameters. The sub-process for selecting the optimisation algorithm is adapted from the work of Tiwari et al. [30]. The user is guided through a series of sequential steps to reveal the nature of the optimisation problem at hand. A number of optimisation algorithms or modules that suit each characteristic are suggested. Nine suitable algorithms are suggested for multi-objective optimisation. Likewise, suitable algorithms are proposed for problems that require global search, include handling constraints, require robust search or include handling uncertainty. If the selected optimisation algorithm is not included in the simulation software package then often programming will be required to connect the simulation model to the optimisation algorithm. If that is not possible the framework will ask the user to modify the selected optimisation algorithm until it becomes applicable in his/her specific situation. If the used optimisation engine provides the required flexibility, optimisation algorithms needs to be set. For example: Genetic Algorithms can have different numbers of populations, generations, cross over and mutation parameters. On the other hand, parameters in Simulated Annealing (SA) include the cooling factor and the initial temperature.

7. Set the simulation optimisation: To prepare for the experiments, simulation parameters need to be set [31]. This includes the number of replications, warm-up time and the run length. High computational expenses reflected in long estimated runtime is a major issue that might appear at this stage for complex systems. Several strategies for reducing the computation time are suggested such as improving the computation speed using parallel computing or grid computing. Alternatively, special optimisation algorithms can reduce the 
computation time significantly. In some cases, there will be a need to go back to previous steps in order to decrease the simulation time by reducing the number of replications or the simulation run-length. Otherwise, the optimisation problem would have to be simplified by minimising the variables' ranges, reducing the number of variables or reducing the number of objective functions if possible. It may be useful to monitor additional parameters that are not defined as objective functions. This is usually defined at this stage in order to have each response recorded with its corresponding solution. At the end of this step the simulation optimisation will be conducted.

8. Decision making: After the optimisation results are produced, they need to be interpreted in light of the current business context. This is particularly important in multiobjective optimisation where one objective might be relatively more important than others depending on business dynamics. Nevertheless, considering the business context is also relevant to single objective optimisation. There might be multiple combinations of decision variables that result in comparative values for the objective function. Likewise, monitored responses might have an effect on the choice of implemented solution. If areas of high uncertainty are identified that are not addressed adequately by stochastic simulation or by special optimisation algorithms then sensitivity analysis is suggested. This can be achieved by investigating which inputs have high uncertainties, followed by defining additional scenarios with the new input values to run the simulation optimisation repeatedly. If no further sensitivity analysis is required, the optimal values can be chosen as the solutions.

\section{DISCUSSION}

Prior reviews in maintenance optimisation have repeatedly reported the need for a framework that provides adequate level of details to guide both academics and practitioners in optimising complex maintenance systems. This study set out with the aim of addressing this gap by developing a framework to guide the process of maintenance optimisation through simulation. In contrast to earlier studies, the proposed framework was developed based on an evaluation of existing frameworks in addition to capturing framework requirements. As illustrated in Table I, existing frameworks seem to stand short of meeting most of the requirements.

Table I: Evaluating maintenance optimisation frameworks against the requirements.

\begin{tabular}{|c|l|c|c|c|}
\hline \multicolumn{1}{|c|}{ Requirements } & Chien et al. [5] & Riane et al. [6] & $\begin{array}{c}\text { Horenbeek et } \\
\text { al. [2] }\end{array}$ & $\begin{array}{c}\text { Proposed } \\
\text { framework }\end{array}$ \\
\hline 1 & $\begin{array}{l}\text { Assist users with typical uncertainty found in maintenance } \\
\text { systems }\end{array}$ & No & Yes \\
\hline 2 & $\begin{array}{l}\text { Assist users to adapt maintenance models to their specific } \\
\text { business needs }\end{array}$ & No & No & Yes \\
\hline 3 & Enable users to solve multi-objective optimisation & No & No & No \\
\hline 4 & Assist users with complex maintenance systems & No & Yes & Yes \\
\hline 5 & $\begin{array}{l}\text { An operational decision making tool suitable for maintenance } \\
\text { managers and practitioners }\end{array}$ & Yes & Yes \\
\hline 6 & Incorporating production dynamics and spare parts management & No & No & Yes \\
\hline 7 & $\begin{array}{l}\text { Allow the investigation of several maintenance strategies } \\
\text { simultaneously }\end{array}$ & No & Yes & Yes \\
\hline 8 & Incorporating possible future maintenance strategies & No & No & No \\
\hline 9 & Integration with e-maintenance & No & Yes & No \\
\hline
\end{tabular}

Uncertainty can be addressed partially by defining stochastic inputs in the simulation model. The proposed framework assists users with typical uncertainty by suggesting specific optimisation algorithms (sub-process 6.1). In addition, specific sources of high uncertainties in maintenance systems are identified so the user can decide if any of them are present in the maintenance system (sub-process 8.1). Throughout the framework, the user is advised on the 
optimisation objectives, decision variables and constraints suitable for the maintenance system in interest. By following the framework steps, the user would have a model that meets his/her specific business needs. If multi-objective optimisation is required, the framework allows the user to formulate the objectives in a systematic way. Furthermore, several multiobjective optimisation algorithms are suggested (sub-process 6.1). It is impractical to optimise numerous components in a complex maintenance system. Therefore, tools and techniques are suggested to select the most critical assets in the maintenance system (subprocess 1.1). Additionally, complex maintenance systems can introduce the risk of high computation expenses. This is dealt with by suggesting various strategies including improving the computation speed, utilising special optimisation algorithms and simplifying the problem (sub-process 7.1). A standard flow chart is utilised to represent the framework since it is familiar to both maintenance managers and academics. The flow chart guides the user starting from defining the scope of the problem to obtaining the solution and interpreting the results in light of the current business environment through a series of steps containing various processes and decision nodes.

The effect of well-documented factors on the maintenance system is considered in this framework. The scope of the optimisation can include production dynamics and spare parts policies based on the user's circumstances (sub-process 1.2). In the second step of the framework, various maintenance strategies and policies are put forward for the user (subprocesses $2.1 \&$ 2.2). Multiple strategies and policies can be selected for each asset including advanced maintenance strategies such as CBM and self-maintenance. The optimisation then will yield the optimum strategy along with its parameters for each asset.

However, it is not possible to integrate the proposed framework in its current form with emaintenance. Software can be developed to suggest inputs as the user progresses from one stage to another. This will make it even easier to apply since only feasible options will displayed. In addition, the data can stream directly from other maintenance data sources such as condition monitoring sensors and maintenance history records to form a comprehensive decision support system.

\section{CONCLUSIONS}

The literature covers a wide range of simulation based optimisation of maintenance systems. This includes a wide range of maintenance strategies and policies, optimisation objectives, decision variables and optimisation algorithms. The purpose of the current study is to develop a simulation based optimisation framework that supports decision making for maintenance in manufacturing systems.

This research extends our knowledge by identifying nine requirements for the framework. The requirements were established by examining review papers in maintenance optimisation as well as publications on future maintenance applications. Furthermore, existing maintenance optimisation frameworks were examined and evaluated against these requirements.

A novel framework was developed to aid future attempts to optimise complex maintenance systems through simulation. A key strength of the proposed framework is its ability to meet most of the requirements. Current issues addressed by the framework include complexity, uncertainty, high computation expenses and advanced maintenance applications.

A future study optimising an empirical maintenance system would be very interesting. In addition, developing a similar framework for simulating maintenance systems would be a fruitful area for further work. The simulation framework can suggest various modelling tools and techniques to the user based on the current maintenance system characteristics and configuration. Finally, a software tool can be developed to enable the proposed framework to integrate with e-maintenance. 


\section{ACKNOWLEDGEMENT}

The authors would like to thank the University of Jeddah for funding this research.

\section{REFERENCES}

[1] Alrabghi, A.; Tiwari, A. (2015). State of the art in simulation-based optimisation for maintenance systems, Computers \& Industrial Engineering, Vol. 82, 167-182, doi:10.1016/j.cie.2014.12.022

[2] Van Horenbeek, A.; Pintelon, L.; Muchiri, P. (2010). Maintenance optimization models and criteria, International Journal of Systems Assurance Engineering and Management, Vol. 1, No. 3, 189-200, doi:10.1007/s13198-011-0045-x

[3] Deb, K. (2005). Optimization for engineering design: algorithms and examples, Prentice-Hall, New Delhi

[4] Cavazzuti, M. (2013). Optimization methods - From theory to design scientific and technological aspects in mechanics, Springer-Verlag, Berlin

[5] Chien, T. W.; Lin, C.; Sphicas, G. (1997). A systematic approach to determine the optimal maintenance policy for an automated manufacturing system, Quality and Reliability Engineering International, Vol. 13, No. 4, 225-233, doi:10.1002/(SICI)1099-1638(199707)13:4<225::AIDQRE106>3.0.CO;2-X

[6] Riane, F.; Roux, O.; Basile, O.; Dehombreux, P. (2009). Simulation based approaches for maintenance strategies optimization, Ben-Daya, M.; Duffuaa, S. O.; Raouf, A.; Knezevic, J.; AitKadi, D. (Eds.), Handbook of Maintenance Management and Engineering, Springer, London, 133-153

[7] Nicolai, R. P.; Dekker, R. (2008). Optimal maintenance of multi-component systems: a review, Kobbacy, K. A. H.; Murthy, D. N. P. (Eds.), Complex system maintenance handbook, Springer, London, 263-286

[8] Dekker, R. (1996). Applications of maintenance optimization models: a review and analysis, Reliability Engineering \& System Safety, Vol. 52, No. 3, 229-240, doi:10.1016/09518320(95)00076-3

[9] Garg, A.; Deshmukh, S. G. (2006). Maintenance management: literature review and directions, Journal of Quality in Maintenance Engineering, Vol. 12, No. 3, 205-238, doi:10.1108/ 13552510610685075

[10] Sharma, A.; Yadava, G. S.; Deshmukh, S. G. (2011). A literature review and future perspectives on maintenance optimization, Journal of Quality in Maintenance Engineering, Vol. 17, No. 1, 525, doi:10.1108/13552511111116222

[11] Van Horenbeek, A.; Buré, J.; Cattrysse, D.; Pintelon, L.; Vansteenwegen, P. (2013). Joint maintenance and inventory optimization systems: a review, International Journal of Production Economics, Vol. 143, No. 2, 499-508, doi:10.1016/j.ijpe.2012.04.001

[12] Vasili, M.; Hong, T. S.; Ismail, N.; Vasili, M. (2011). Maintenance optimization models: a review and analysis, Proceedings of the 2011 International Conference on Industrial Engineering and Operations Management, 1131-1138

[13] Hong, D. S.; Kamaruddin, S.; Azid, I. A. (2012). Maintenance policy selection: a review towards building proper selection model, International Journal of Industrial and Systems Engineering, Vol. 10, No. 3, 355-375, doi:10.1504/IJISE.2012.045680

[14] Ding, S.-H.; Kamaruddin, S. (2015). Maintenance policy optimization - literature review and directions, The International Journal of Advanced Manufacturing Technology, Vol. 76, No. 5-8, 1263-1283, doi:10.1007/s00170-014-6341-2

[15] Lee, J.; Ghaffari, M.; Elmeligy, S. (2011). Self-maintenance and engineering immune systems: Towards smarter machines and manufacturing systems, Annual Reviews in Control, Vol. 35, No. 1, 111-122, doi:10.1016/j.arcontrol.2011.03.007

[16] Muller, A.; Crespo Marquez, A.; Iung, B. (2008). On the concept of e-maintenance: Review and current research, Reliability Engineering \& System Safety, Vol. 93, No. 8, 1165-1187, doi:10.1016/j.ress.2007.08.006 
[17] Jantunen, E.; Emmanouilidis, C.; Arnaiz, A.; Gilabert, E. (2011). E-Maintenance: Trends, challenges and opportunities for modern industry, Proceeding of the $18^{\text {th }}$ IFAC World Congress, 453-458

[18] Starr, A.; Al-Najjar, B.; Holmberg, K.; Jantunen, E.; Bellew, J.; Albarbar, A. (2010). Maintenance today and future trends, Holmberg, K.; Adgar, A.; Arnaiz, A.; Jantunen, E.; Mascolo, J.; Mekid, S. (Eds.), E-maintenance, Springer, London, 5-37

[19] Ojanen, V. (2014). Maintenance innovations - Types, patterns and emerging trends, 2014 IEEE International Conference on Management of Innovation and Technology, ICMIT 2014, 321-326

[20] Campos, J. (2014). Current and prospective information and communication technologies for the e-maintenance applications, Journal of Quality in Maintenance Engineering, Vol. 20, No. 3, 233248, doi:10.1108/JQME-05-2014-0029

[21] Liyanage, J. P. (2010). State of the art and emerging trends in operations and maintenance of offshore oil and gas production facilities: Some experiences and observations, International Journal of Automation and Computing, Vol. 7, No. 2, 137-145, doi:10.1007/s11633-010-0137-0

[22] Ao, Y. H. (2011). A review on development and trend of Intelligent Maintenance System, Advanced Materials Research, Vol. 314-316, 2365-2369, doi:10.4028/www.scientific.net/ AMR.314-316.2365

[23] Marais, K. B.; Robichaud, M. R. (2012). Analysis of trends in aviation maintenance risk: An empirical approach, Reliability Engineering \& System Safety, Vol. 106, 104-118, doi:10.1016/ j.ress.2012.06.003

[24] Sharma, B.; Arora, A. K.; Kumar, P. (2010). Modern trends in application, installation, operation \& maintenance of large power transformers, Water and Energy International, Vol. 67, No. 1, 4958

[25] Gomes, W. J. S.; Beck, A. T.; Haukaas, T. (2013). Optimal inspection planning for onshore pipelines subject to external corrosion, Reliability Engineering \& System Safety, Vol. 118, 18-27, doi:10.1016/j.ress.2013.04.011

[26] Belegundu, A. D.; Chandrupatla, T. R. (2011). Optimization concepts and applications in engineering, Cambridge University Press, Cambridge

[27] Lei, Y.; Liu, J.; Ni, J.; Lee, J. (2010). Production line simulation using STPN for maintenance scheduling, Journal of Intelligent Manufacturing, Vol. 21, No. 2, 213-221, doi:10.1007/s10845008-0194-1

[28] Bruzzone, A.; Bocca, E. (2012). Innovative solutions based on simulation combined with optimization techniques for maintenance service management within complex systems, International Journal of Modeling, Simulation, and Scientific Computing, Vol. 3, No. 2, 23 pages, doi: $10.1142 / \mathrm{S} 1793962312400041$

[29] Laggoune, R.; Chateauneuf, A.; Aissani, D. (2009). Opportunistic policy for optimal preventive maintenance of a multi-component system in continuous operating units, Computers \& Chemical Engineering, Vol. 33, No. 9, 1499-1510, doi:10.1016/j.compchemeng.2009.03.003

[30] Tiwari, A.; Hoyos, P. N.; Hutabarat, W.; Turner, C.; Ince, N.; Gan, X.-P.; Prajapat, N. (2015). Survey on the use of computational optimisation in UK engineering companies, CIRP Journal of Manufacturing Science and Technology, Vol. 9, 57-68, doi:10.1016/j.cirpj.2015.01.003

[31] Robinson, S. (2004). Simulation: The practice of model development and use, Wiley, Chichester

\section{APPENDIX A}

The third level of the framework: https://goo.gl/nfDyoG 